\title{
Two alternative models concerning the perialveolar microcirculation in mammalian lungs
}

\author{
BRUNO GÜNTHER $^{a}$, ENRIQUE MORGADO ${ }^{\mathrm{b}, \mathrm{c} *}$ and MANUELA COCIÑ $\mathrm{A}^{\mathrm{d}}$
}

\author{
${ }^{a}$ Professor Emeritus of Physiology and Pathophysiology, University of Chile, University of Concepción, and \\ University of Valparaíso \\ b Pathophysiology Program, Institute of Biomedical Sciences, Faculty of Medicine, University of Chile \\ ${ }^{c}$ Pathophysiology Program, Faculty of Medical Sciences, University of Santiago of Chile \\ ${ }^{\mathrm{d}}$ Faculty of Medicine, University of Chile
}

\begin{abstract}
Despite the fact that the concept of sheet-flow in the pulmonary microcirculation of mammals was introduced more than three decades ago, the capillary circulatory model still prevails in the physiological literature. Since cardiac output is identical in the systemic and in pulmonary circulations, it is noteworthy that in the former, the resulting arterial pressure is five times higher than that of the latter, which means that the corresponding microcirculations must be radically different. The present study addresses this problem from both morphological and physiological perspectives.
\end{abstract}

Key terms: arterial pressures, capillaries, pulmonary microcirculation, sheet flow, systemic circulation

\section{INTRODUCTION}

In the pertinent literature and invariably in all textbooks concerning the rheology of blood in mammalian lungs, the terminal branches of the pulmonary circulation are described as "capillaries". However, the process of gas diffusion of either $\mathrm{O}_{2}$ or $\mathrm{CO}_{2}$ at the alveolar level could be enhanced to a maximum if the flow of blood were spread out into a very thin layer at the surface of each alveolar sac. Since the primary function of the lungs is to oxygenate the blood and remove $\mathrm{CO}_{2}$, the morphology and physiology at the air-blood interface is of paramount importance, as will be discussed in two alternative models. The aim of the present study is to compare the systemic with the pulmonary circulation in mammals, since in both cases the cardiac output percolates through a maze of microvascular channels connected in series.
The first case deals with true capillaries of the systemic vascular system, while the second case pertains to a thin sheet of blood that flows around pulmonary alveoli.

\section{The capillary flow model}

In 1681 Marcello Malpighi (1628-1694) coined the term "capillary," a neologism derived from the Latin capillus = hair, to describe the microcirculation he discovered in slightly inflated frog lungs while examining them with a double convex lens (see Fig. 1A). The quantitative analysis of the blood flow in cylindrical tubes can be performed by means of the classical Poiseuille's (1828) formula, which reads as follows:

$$
Q=\frac{\pi \cdot r^{4}}{8 \cdot \eta \cdot l} \cdot \Delta \mathrm{P}
$$


Where:

$\boldsymbol{Q}=$ total volume of liquid flowing through a tube per unit of time;

$r=$ radius of the cylindrical tube;

$\eta=$ dynamic viscosity of the fluid;

$l=$ length of the tube; and

$\Delta \mathrm{p}=$ pressure difference along a cylindrical vessel

The corresponding dimensional analysis of equation (1) reads as follows:

$\mathrm{Q}=\left(\left[\mathrm{L}^{4}\right] \cdot\left[\mathrm{M}^{1} \cdot \mathrm{L}^{-1} \cdot \mathrm{T}^{-2}\right]\right) /\left(\left[\mathrm{M}^{1} \cdot \mathrm{L}^{-1} \cdot \mathrm{T}^{-1}\right] \cdot\left[\mathrm{L}^{1}\right]\right)=\left[\mathrm{L}^{3} \cdot \mathrm{T}^{-1}\right]$
However, eqn. 1 is only valid for uniform Newtonian fluids of constant viscosity without elements in suspension such as erythrocytes, leucocytes, or platelets, and provided that the flow is laminar (parabolic velocity profile). Furthermore, the geodesic of each microvessel should be at least equivalent to ten times the diameter of the corresponding cylindrical tubing. In the case of blood, the Fahraeus-Lindquist (1931) effect is apparent for cylindrical tubes of diameters less than $0.8 \mathrm{~mm}$ (terminal arteries,
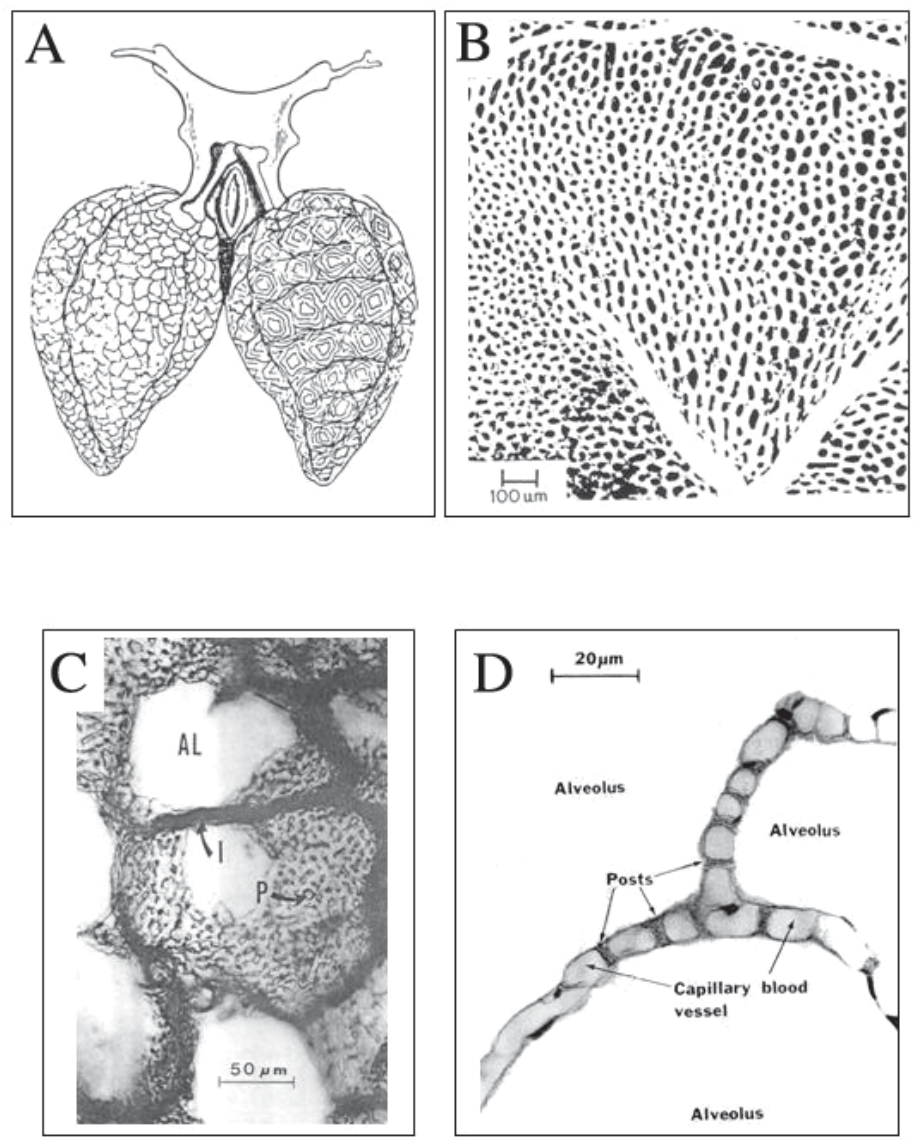

Figure 1A: Original drawing by Marcello Malpighi (1681): preparation of two slightly inflated frog lungs, where a reticulum of microvessels is apparent in place of "capillaries". (Adapted from Fishman and Richards, 1964).

Figure 1B: Photograph of a network of blood vessels in the alveolar wall (Fung, 1984).

Figure 1C: Pulmonary microcirculation in the cat. $\mathrm{AL}=$ alveolar air spaces. $\mathrm{P}$ = post. $\mathrm{I}=$ interalveolar septum (Fung, 1984).

Figure 1 D: Three interalveolar septum. (Fung, 1984) 
arterioles, precapillaries and capillaries), converting blood into a non-Newtonian fluid.

The Bingham effect (McDonald, 1974), which specifically deals with variable viscosity, should also be mentioned as an additional anomaly because some materials behave as solids when the shear rate is zero, while the relationship between the shear rate and the shear stress is linear above a given value of shear stress.

With regard to the Reynolds number Re (laminar vs. turbulent flow) in the case of microcirculation, $\mathrm{Re}=0.03$ in the arterioles, and $\mathrm{Re}=0.0025$ in the systemic capillaries, in contrast with the blood flow in the aorta, where $\operatorname{Re}=2349$ (Withers, 1992).

Also worthy of note is the fact that the microcirculation of all mammalian species, from the 3-gram shrew to the 3 -ton elephant, can be characterized by some invariant features, such as the length of all mammalian capillary vessels, which is approximately $1 \mathrm{~mm}$ (West et al. 1997, 1999). On the other hand, the capillary diameters correlate to the maximal diameters of the prevalent red blood cells (7-8 $\mu \mathrm{m})$.

\section{The sheet-flow paradigm}

The blood flow in the pulmonary microcirculation of mammals differs from the classical systemic circulation in the sense that instead of a set of parallel cylindrical capillaries, the perialveolar blood flow is limited by two separated endothelial sheets whose distance remains almost constant $(3.5 \mu \mathrm{m})$ due to the presence of an array of cylindrically-shaped "posts" (Fung and Sobin, 1969, 1972; Sobin et al., 1970; Fung, 1981, 1984; see Figure 1 $\mathrm{B}, \mathrm{C}, \mathrm{D})$. In consequence, this interstitial sheet-flow model of circulation, also known as a "porous medium," depends upon "permeability" (hydraulic conductivity), which is determined by the ratio between the relative cross-sectional area of the vascular spaces (v) and the tissue spaces (t) at a given transsection of the alveolar wall. The volume-flow varies directly with the pressure drop per unit length and inversely with viscosity. In addition to the porosity or the permeability of the bed $(\mathrm{v} / \mathrm{t})$, it is necessary to take into account the tortuosity of these channels, since instead of the almost linearity of the blood flow in the systemic capillaries, in the sheet-flow, blood follows a sinusoidal trajectory due to the alternative position of the cylindrical post. Thus, the sheet-flow model is in agreement with Darcy's equation (1856):

$$
Q=\frac{v}{t} \cdot \frac{1}{\eta} \cdot \Delta \mathrm{P}
$$

Where:

$Q=$ the total volume of liquid flowing through the porous medium per unit time,

$\mathrm{v}=$ cross-sectional area of the vascular space,

$t=$ cross-sectional area of the tissue space (post), conformed by microcylinders of elastin and

$\mathrm{q}=$ the volume of blood contained in the microvascular system which characterizes the geometric sheet configuration

$\eta=$ dynamic viscosity of the fluid medium, and

$\Delta \mathrm{P}=$ pressure drop

The corresponding dimensional analysis reads:

$\mathrm{Q}=\left(\left[\mathrm{L}^{2}\right] \cdot\left[\mathrm{L}^{3}\right] \cdot\left[\mathrm{M}^{1} \cdot \mathrm{L}^{-1} \cdot \mathrm{T}^{-2}\right]\right) /\left(\left[\mathrm{L}^{2}\right] \cdot\left[\mathrm{M}^{1} \cdot \mathrm{L}^{-1} \cdot \mathrm{T}^{-1}\right]\right)=\left[\mathrm{L}^{3} \cdot \mathrm{T}^{-1}\right]$

Furthermore, Darcy's law is a lowReynolds number relationship of interstitial flow of a porous medium (sheet-flow), which was studied by French hydraulic engineer H. Darcy in 1856 a few years after Poiseuille's law (1826) was published.

\section{DISCUSSION}

The quantitative analysis of blood flow in vascular territories of the microcirculation finally yielded two equations; one is the well-known Poiseuille formula, and the other is Darcy's sheet-flow equation. In both instances the liquid should be one of 
Newtonian characteristics, of uniform viscosity and without particles in suspension. Furthermore, and in accordance to the Fahraeus-Lindquist (1931) phenomenon, the flow of blood at $37^{\circ} \mathrm{C}$ with a hematocrit of $40-45 \%$ and viscosity expressed in centipoises is a function of the diameter of the capillary vessel. Thus the relative blood viscosity is markedly reduced when the vessel diameter is less than 0.8 $\mathrm{mm}$, whereas plasma and saline solution do not change their viscosity under these circumstances. In consequence, neither the condition of a constant viscosity in both types of flow nor the Bingham effect have been taken into account (Vogel, 1981, 2003).

The fractal nature of the respiratory and the circulatory systems has been analyzed by Weibel et al (1962), and by Weibel (1984, 2000) for 23 generations of airways and to 28 generations of pulmonary circulation in cats up to the capillary level, whereas in the sheet-flow level, the pulmonary perialveolar microcirculation is represented by a meshwork of approximately 160 hexagonal units per alveolar sac formed by two endothelial sheet connected by an array of cylindrical posts. In cat lung (Fung, 1984) with a blood pressure gradient $\Delta \mathrm{P}=10.3 \mathrm{cmH}_{2} \mathrm{O}$, each hexagon occupies an area of $202 \mu \mathrm{m}^{2}$, while the total surface area of one alveolus of 100 $\mu \mathrm{m}$ is equivalent to $31416 \mu^{2}$. Assuming that the hexagonal microvascular units have a similar area in humans, Weibel (2000) estimated that the terminal alveolar sacs have a diameter of approximately $250 \mu \mathrm{m}$, in which case the number of hexagons per alveolus would be 972 (Weibel, 2000).

Despite the fact that the blood flow (cardiac output), in both the systemic and the pulmonary systems is the same provided steady-state hemodynamic conditions prevail, the mean systemic arterial pressure is $100 \mathrm{mmHg}$ while the mean pulmonary pressure is $20 \mathrm{mmHg}$. This pronounced difference $(5 / 1)$ is attributed to the respective total peripheral resistances (TPR), which in the former case was the resistance of terminal arteries, precapillaries, and capillaries of the systemic circulation and the resistance of the sheet-flow in the latter.
For the correlation between form and function in mammalian lung, Taylor and Weibel (1981), and Weibel (2000) coined the term "symmorphosis," which was derived from the Greek ovv, which means "balanced formation" and morwsi", which is correlated with form or figure. According to these authors, the concept is associated with 1) economic design, 2) adaptation of structure to functional needs, and 3) integration of the part into the whole. However, from a strictly etymological perspective, this neologism seems to emphasize the morphological aspects rather than the corresponding functions. Another possibility might be the integrative concept of beltistometrics, a term also of Greek origin, derived from bevtisto: optimal solution, and metron measure; in sum, a quantitative evaluation of the best solution for a given problem.

The sheet flow model of the pulmonary microcirculation in all mammals represents:

1) the maximal economy of cellular elements;

2) the bilateral exposure of red cells to the oxygen content of alveolar air (see Fig. 1 D);

3) a minimal and constant diffusion distance between the air within the alveolar sacs and the hemoglobin inside each erythrocyte, due to the existence of inter-membrane posts;

4) a sinusoidal blood flow trajectory, which increases the length and diffusion time of the perialveolar network of the microcirculation (see Fig. 2);

5) an accidental obstruction of the normal pathway due to microthrombosis or embolism will not affect the primary pulmonary circulation due to the existence of nearby alternative pathways;

6) in contrast with the results of Poiseuille's rule, the total resistance (TR) pertaining to the sheet flow model is equivalent to $1 / 5$ of the hindrance represented by the capillary model when applied to the blood flow in cylindrical microvessels.

In conclusion, the "oxygen cascade" consists of two bulk transports in series 

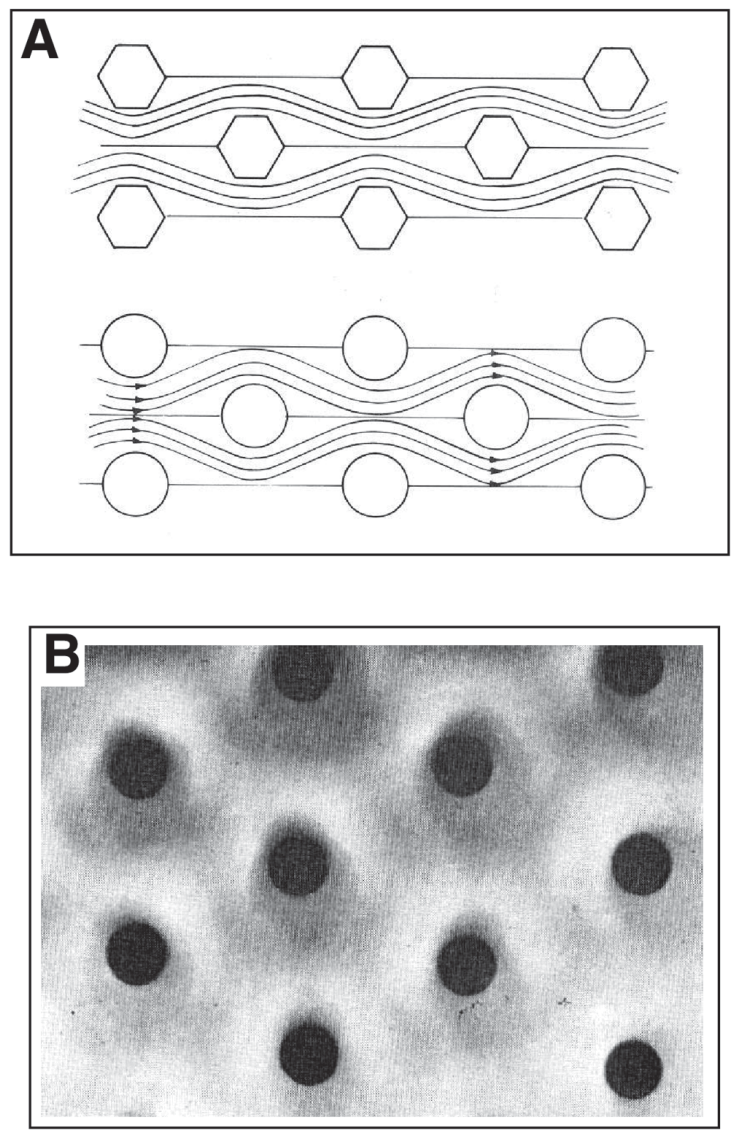

Figure 2A: Sinusoidal streamlines of blood flow in a network of hexagonal and cylindrical posts of a sheet flow model (Fung, 1984).

Figure 2B: Two sheets of rubber glued together at post areas and inflated by air and with a constant vascular space-tissue ratio (Fung and Sobin, 1972).

(respiration and circulation) and two diffusion barriers at the lungs and tissues, where the corresponding microcirculations (sheet-flow and capillary flow) are the exchange areas of the low pressure and high pressure arterial circulations. The two models of diffusion transport (sheet and capillary), which also function in series, are representative of "optimal solutions," from both morphological and physiological perspectives, as was defined by the two neologisms, symmorphosis and beltistometrics. These conclusions are valid for all terrestrial mammals, regardless of size.

In accordance with the Darwinian principles of natural selection, low pressure vascular resistance should prevail because it minimizes the matter and energy requirements per unit of time. However, the high pressure in the systemic arterial tree accomplishes other vital functions, such as the renal excretion of catabolites and the transfer of the plasma components between the blood compartment and the interstitial fluid. In contrast, low-pressure and low resistance pulmonary microcirculation, where the colloidosmotic pressure is higher than the corresponding hydrostatic pressure, is compensated by means of the negative interstitial pressure in order to assure normal lymphatic drainage (Guyton, 1991). 
In the pathological realm, it is worth mentioning that pulmonary arterial pressure rises when there is an increment of the corresponding arteriolar (pre-capillary) or venular resistance (post-capillary), however, these abnormalities are caused by the cylindrical blood vessels, and are in accordance with Poiseuille's formula.

The sheet-flow design of the respiratory microvascular system of complex animals has been analyzed thoroughly by Maina (2000) from a morphological and physiological perspective, with emphasis on the fractal nature of mammalian lungs as well as the low-pressure and low-resistance characteristics, yielding optimal gas diffusion and minimal structural failures as engineered by natural selection and genomically conserved. The integrative design strategy for these gas exchangers in porous media enabled the respiratory system to respond adequately to oxygen consumption, which can vary from resting to maximal exercise conditions, an adaptive effort that comprises a scale from one to ten.

The perialveolar sheet-flow model could be compared with the classical capillary-flow model on the basis of Poiseuille's formula and in contrast with D'Arcy equation, which concerns the two endothelial sheets separated by a set of alternative posts that determine a sinusoidal pathway of the perialveolar microcirculation rather than a linear or geodesic trajectory of the blood cells on the surface of each pulmonary alveolus. These sinusoidal a-v trajectories should be associated with a greater drop in arterialvenous pressure and turbulence around the above-mentioned post. This does not happen, however, because blood's viscosity is higher than that of water and the extremely low Reynolds number $(\operatorname{Re}<0.0025)$.

\section{REFERENCES}

DARCY H (1856) Les fontaines publiques de la ville de Dijon. Paris: Dalmont

FAHRAEUS R, LINDQUIST T (1931) The viscosity of the blood in narrow capillary tubes. Am J Physiol 96: 562568

FISHMAN AP, RICHARDS DW (Eds.) (1964) Circulation of Blood. Men and Ideas. New York: Oxford University Press

FUNG YC, SOBIN SS (1969) Theory of sheet flow in lung alveoli. J Appl Physiol 26: 472-488

FUNG YC, SOBIN SS (1972) Elasticity of the pulmonary alveolar sheet. Circ Res 30: 451-469

FUNG YC (1981) Biomechanics. Mechanical Properties of Living Tissues. New York: Springer.

FUNG YC (1984) Biodynamics. Circulation. New York: Springer

GUYTON AC (1991) Textbook of Medical Physiology. $8^{\text {th }}$ ed., Philadelphia: Saunders

MAINA JN (2000) Is the sheet-flow design a 'frozen core' (a Bauplan) of the gas exchangers? Comparative functional morphology of the respiratory microvascular systems: illustration of the geometry and rationalization of the fractal properties. Comp Biochem Physio Part A 126: 491-515

MCDONALD DA (1974) Blood Flow in Arteries Baltimore: Williams \& Wilkins

SOBIN SS, TREMER HM, FUNG YC (1970) Morphometric basis of the sheet-flow concept of the pulmonary alveolar microcirculation in the cat. Circulation Res 26: 397-414

TAYLOR CR, WEIBEL ER (1981) Design or the mammalian respiratory system. I. Problems and strategy. Respir Physiol 44: 1-10

VOGEL S (1981) Life in Moving Fluids. The Physical Biology of Flow. Boston: Willard Grant Press

VOGEL S (2003) Comparative Biomechanics. Life's Physical World. Princeton: Princeton University Press

WEIBEL ER, GÓMEZ DM (1962) Architecture of the human lung. Sci 137: 577-585

WEIBEL ER (1984) The Pathway for Oxygen. Structure and Function in the Mammalian Respiratory System. Cambridge, MA: Harvard University Press

WEIBEL ER (2000) Symmorphosis. On Form and Function in Shaping Life. Cambridge, MA: Harvard University Press

WEST GB, BROWN JH, ENQUIST BJ (1997) A general model for the origin of allometric scaling laws in biology. Sci 276: 122-126

WEST GB, BROWN JH, ENQUIST BJ (1999) The fourth dimension of life: fractal geometry and allometric scaling of organisms. Sci 284: 1677-1679

WITHERS PC (1992) Comparative Animal Physiology. Forth Worth: Saunders 